\title{
Association between obesity and iron deficiency (Review)
}

\author{
NASEEM MOHAMMAD ALSHWAIYAT ${ }^{1}$, ARYATI AHMAD ${ }^{1,2}$ \\ WAN MOHD RAZIN WAN HASSAN ${ }^{3}$ and HAMID ALI NAGI AL-JAMAL ${ }^{4}$
}

\footnotetext{
${ }^{1}$ School of Nutrition and Dietetics, Faculty of Health Sciences, Universiti Sultan Zainal Abidin, Gong Badak Campus, Kuala Terengganu, Terengganu 21300, Malaysia; ${ }^{2}$ Nuffield Department of Primary Care Health Sciences, University of Oxford, Radcliffe Primary Care Building, Radcliffe Observatory Quarter, Oxford OX2 6GG, UK; ${ }^{3}$ Faculty of Medicine, Universiti Sultan Zainal Abidin, Medical Campus, Kuala Terengganu, Terengganu 20400;

${ }^{4}$ School of Biomedicine, Faculty of Health Sciences, Universiti Sultan Zainal Abidin, Gong Badak Campus, Kuala Terengganu, Terengganu 21300, Malaysia
}

Received April 28, 2021; Accepted July 22, 2021

DOI: $10.3892 / \mathrm{etm} .2021 .10703$

\begin{abstract}
Obesity is a risk factor for several comorbidities and complications, including iron deficiency anemia. Iron deficiency anemia is a serious global public health problem, with a worldwide prevalence. The high prevalence of obesity in combination with iron deficiency incidence observed in different age and sex categories suggests an association between obesity and iron status. Obesity may disrupt iron homeostasis, resulting in iron deficiency anemia. The association between obesity and iron deficiency may be due to increased hepcidin levels mediated by chronic inflammation. Hepcidin is a small peptide hormone that functions as a negative regulator of intestinal iron absorption. Significant body weight loss in overweight and obese individuals decreases chronic inflammation and serum hepcidin levels, resulting in improved iron status due to increased iron absorption. However, further randomized controlled trials are required to confirm this effect.
\end{abstract}

\section{Contents}

1. Introduction

2. Iron metabolism

3. Hepcidin and iron homeostasis

4. Obesity and low-grade chronic inflammation

5. Association between overweight and obesity, and iron status

Correspondence to: Dr Hamid Ali Nagi Al-Jamal, School of Biomedicine, Faculty of Health Sciences, Universiti Sultan Zainal Abidin, 2nd Floor, Maimunah Block, Gong Badak Campus, 174 Jalan Sultan Mahmud, Kuala Terengganu, Terengganu 21300, Malaysia E-mail: aljamalhamid@unisza.edu.my

Key words: anemia, chronic inflammation, hepcidin, iron deficiency, obesity, weight loss
6. Effect of weight loss on iron status

7. Future research and clinical implications

8. Conclusions

\section{Introduction}

Obesity and iron deficiency are global health problems affecting billions of people worldwide $(1,2)$. While overweight and obesity are the key risk factors for many chronic diseases, such as cardiovascular diseases, diabetes, and certain cancers (3), iron deficiency or hypoferremia is the most prevalent single micronutrient deficiency globally (4). Untreated iron deficiency can cause iron deficiency anemia, a severe health problem that appears in the form of tiredness, reduced life productivity, and poor maternal health, especially among pregnant women (5-8). Growing evidence supports the existence of an association between obesity and iron deficiency (9). This link was observed among children, adolescents, and adults (10-12). An observational study of 619 women aged 20-49 years has reported that iron deficiency was identified in $23.5,41.9$, and $45.6 \%$ of women with normal weight, overweight and obesity, respectively (13). Another study revealed that iron deficiency (serum iron $<60 \mu \mathrm{g} / \mathrm{dl}$ ) was detected in $13.5,13.6,23.5$, and $21.7 \%$ of male adolescents $(n=772)$ with underweight, normal weight, overweight and obesity, respectively (14). Egwurugwu et al (2018) reported that mean serum iron was $72.6,64.2,59.1$, and $54.7 \mu \mathrm{g} / \mathrm{dl}$ in adult men with normal weight, overweight, grade 1 obesity and grade 2 obesity, respectively. In contrast, it was 61.2, 52.9, 44.8 , and $39.6 \mu \mathrm{g} / \mathrm{dl}$ in the adult women (15). It is believed that hepcidin level and low-grade chronic inflammation play a central role in the relationship between obesity and hypoferremia (16). Although obesity is recognized as an emerging risk factor for iron deficiency, mechanisms of the relationship are still debatable (17). This review discusses the current evidence on the relationship between obesity and iron deficiency, focusing on factors that influence iron homeostasis, such as hepcidin and low-grade chronic inflammation. Evidence from relevant published experimental articles on iron deficiency and 
obesity published from January 2015 to January 2021 were retrieved by using Scopus, PubMed and Google Scholar and reviewed. Indeed, few reviews have been carried out to explore the relationship between obesity and iron deficiency. However, the previous works have not described the role of hepcidin and low-grade chronic inflammation in iron homeostasis.

\section{Iron metabolism}

Iron is an essential trace element found in many foods, especially red meats. It is a crucial nutrient for optimal physical and cognitive development of the human body (18). The adult human body contains two to four grams of iron, about two-thirds of which is usually stored in hemoglobin, and the remaining (30-40\%) can be found in the iron-binding proteins, such as ferritin and transferrin (19). Iron is mainly utilized in the bone marrow to synthesize the heme part of the hemoglobin to produce red blood cells in a process called erythropoiesis (20). The principal source of iron in this process comes from the recycling of heme iron from ageing red blood cells, which is mediated by macrophage. This process provides about $90 \%$ of the body iron requirement (21). The remaining $10 \%$ should be acquired from the daily diet to replace usual body iron losses (22). The absorption of dietary iron mainly occurs in the intestinal enterocytes in the duodenum and proximal jejunum (23). Ferroportin is the only known iron transporter found in human intestinal enterocytes (24). It transports iron across the basolateral membrane of intestinal enterocytes into the bloodstream, where iron is carried by transferrin and circulated throughout the body (22). Iron-requiring body cells, including red blood cells, express transferrin receptor on their surface to obtain the iron they need. When iron is required, cellular transferrin receptor transcription is elevated to increase iron uptake and vice versa (25). In healthy individuals, the body stores excess iron in ferritin in the liver that can be released into circulation when needed (21).

\section{Hepcidin and iron homeostasis}

Hepcidin is a small peptide hormone that is considered a key regulator for body iron homeostasis (26). Hepcidin is synthesized mainly in the liver and produced at low levels in other organs like adipose tissue (27). Hepcidin regulates plasma iron level by binding to ferroportin leading to internalization and degradation of ferroportin through blockage of cellular iron transport (28). Consequently, dietary iron absorption from the small intestine is downregulated, and thus, serum iron concentration is dropped (29). In addition, hepcidin slows down the release of recycled iron by macrophages to peripheral and iron mobilization from iron stores in the liver or spleen (30) (Fig. 1).

\section{Obesity and low-grade chronic inflammation}

Obesity is associated with low-grade chronic inflammation (31). Several pro-inflammatory cytokines are secreted by adipose tissues, including interleukin- 6 and tumor necrosis factor alpha. Indeed, about one-third of interleukin- 6 in the circulation is released from adipose tissue (32). The principal mechanism that links obesity and iron deficiency is low-grade systemic inflammation, observed in people with obesity (33).
In people with overweight and obesity, serum hepcidin and serum interleukin-6 are significantly higher than those with normal weight $(12,34)$. Hepcidin which is synthesized in the liver is stimulated by pro-inflammatory cytokines such as interleukin-6 (35) (Fig. 2). A recent study reported that overweight and obese women with central adiposity demonstrated higher serum hepcidin, higher inflammation level, lower iron status, and lower iron absorption when fed with supplemental iron (36).

In response to infection and inflammation, the acute phase restricts the iron availability to pathogens by sequestering iron within macrophages resulting in iron deficiency (37). A vital component of the immune system called toll-like receptors (TLR) can identify pathogen-associated molecular patterns (PAMP). Activation of TLR causes iron deficiency primarily through increasing the hepatic hepcidin (38). This situation induces ineffective erythropoiesis, which leads to anemia of inflammation, a kind of anemia linked to infectious diseases, cancer, and chronic kidney disease (39). Several studies have proven that hypoferremia activates transcription of the osteocyte-secreted protein fibroblast growth factor 23 (FGF23) (40). FGF23, a phosphate and vitamin D homeostasis regulator produced from bone (41), is secreted as pro-inflammatory cytokines in response to the activation of TLR4, followed by up-regulation of hepcidin and down-regulation of erythropoietin expression in addition to reduced serum iron and transferrin saturation (42). Contrarily, inhibition of FGF23 signalling alleviates hypoferremia and attenuates dysregulation of erythropoiesis in acute inflammation (43). The link between FGF23 and inflammation is bound to two mechanisms; i) indirect pathway through elevated hepcidin production, resulting in iron sequestration in macrophages and subsequent functional iron deficiency; ii) direct pathway by stimulating HIF1 $\alpha$ transcription leading to increased HIF1 $\alpha$, which together with HIF1 $\beta$ will bind to HIF response element on the FGF23 promoter inducing its transcription (40). Overall, inflammation, iron status, and erythropoietin are known to regulate FGF23 reciprocally $(42,43)$.

\section{Association between overweight and obesity, and iron status}

The first report of a potential connection between people with obesity and iron status appeared in the early 1960s (44). Four decades later, a cross-sectional study conducted in 2003 described a higher prevalence of iron deficiency in overweight and obese children and adolescents (10). Using data from the National Health and Nutrition Examination Survey (NHANES III), a study confirmed that American children with overweight were twice as likely to be iron deficient than children with normal weight (11). Similar findings were also reported among adults. Lecube et al (2006) found that postmenopausal women with obesity had higher soluble transferrin receptor than non-obese postmenopausal women (45). Similarly, Yanoff et al (2007) reported an increase in the prevalence of iron deficiency in obese adults with significantly lower serum iron level and higher soluble transferrin receptor level than non-obese adults (12). In another study, Menzie et al (2008) found a significantly lower level of serum iron and transferrin saturation in adults with obesity when 

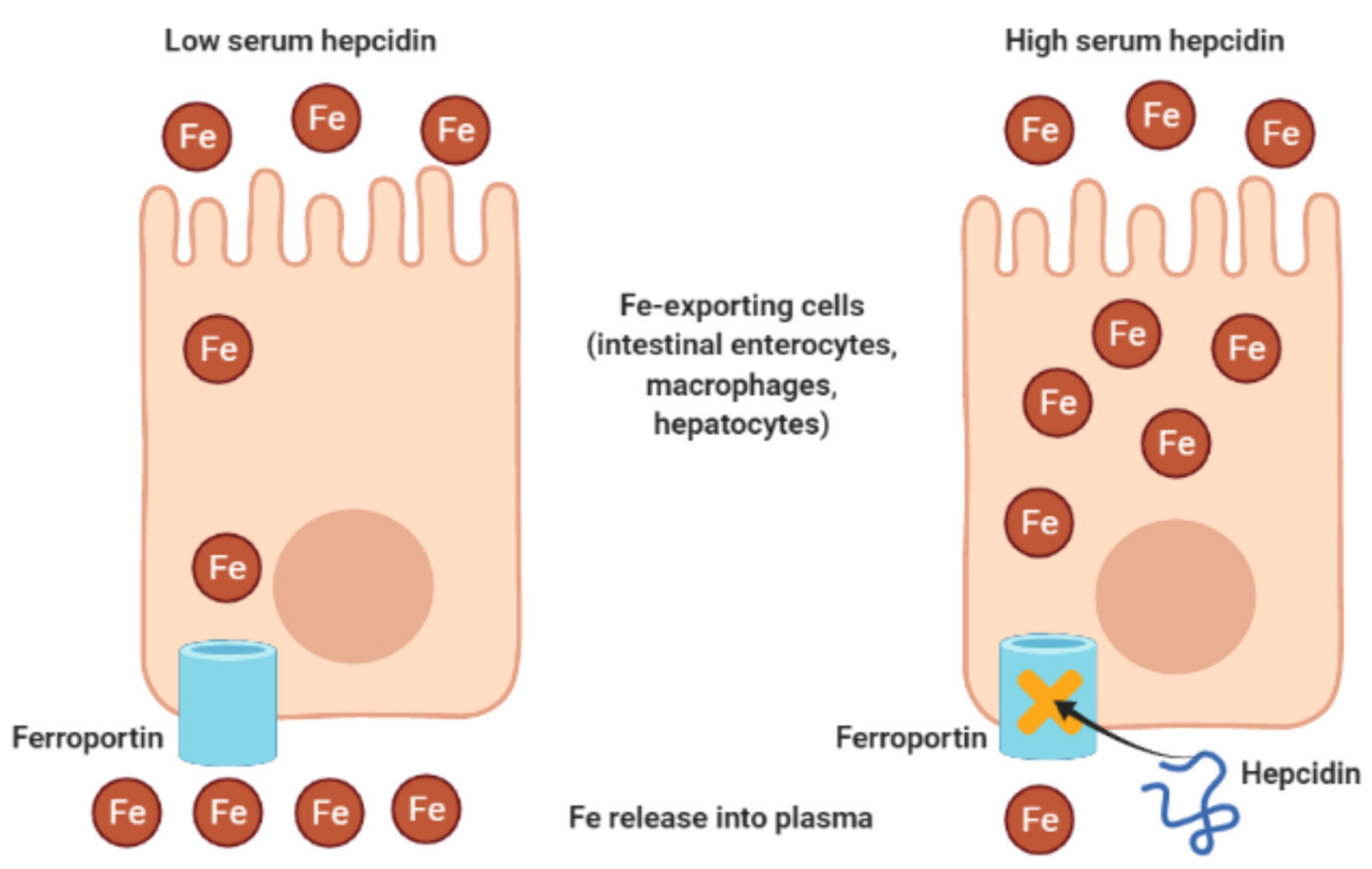

Created in BioRender.com bio

Figure 1. Role of hepcidin in Fe homeostasis. Hepcidin regulates plasma Fe levels by binding to ferroportin, which internalizes and degrades ferroportin by blocking cellular Fe transport.

compared to non-obese adults. In addition, fat mass was found as a significant negative predictor of serum iron concentration (46).

Most studies that explore the relationship between obesity and iron deficiency assessed mainly specific hematological and biochemical markers, such as serum iron, hemoglobin, hematocrit, and ferritin, to evaluate iron status. On the other hand, inflammatory markers and serum hepcidin, which have an essential role in iron homeostasis, are less commonly investigated (47). Paknahad et al (2008) found that serum hemoglobin and hematocrit increased significantly across body mass index (BMI) quartiles in adult Iranian women (48). However, no significant difference in BMI was reported among lactating Kenyan women with different levels of iron depletion (as defined by ferritin), despite hemoglobin was shown to increase with increasing BMI (49). In another report, hematocrit increased with increasing BMI and waist-hip ratio in Nigerian women (50). Aderibigbe et al (2011) showed that waist circumference and waist-hip ratio increased with increasing ferritin concentration in South African women, while serum iron decreased with increasing BMI in women (51). In another study, a lower odds ratio of anemia was reported in women with overweight and obesity than women with normal weight from Egypt and Peru but not from Mexico (52). Surprisingly, Kordas et al (2013) investigated the association between iron deficiency, anemia, and weight status among nonpregnant Colombian women and reported that women with overweight and obesity had a lower likelihood of anemia (53).

A better understanding of the relationship between obesity and iron deficiency has been gained when researchers started to measure inflammatory markers and serum hepcidin in addition to various iron status markers (54). Zimmermann et al (2008) studied the relationship between obesity and iron deficiency among healthy premenopausal women from transition countries using iron isotope labelled test meals and hypothesized that the mechanism might be mediated by inflammation. In their study, $22 \%$ of the women were considered overweight, and $20 \%$ were iron deficient. They measured the iron status indicators (hemoglobin and serum ferritin) and C-reactive protein (CRP) at pre-meal and two-week post-meal. It was observed that fractional iron absorption was negatively correlated with CRP and BMI (55). Alam et al (2015) reported that serum iron and transferrin saturation were significantly lower in individuals with obesity than those with normal body weight, whilst BMI was positively correlated with serum CRP (56). Similarly, Kaner et al (2016) investigated the relationship between iron deficiency and obesity in Turkish women with overweight and obesity and reported that the iron deficiency risk could be more likely to occur in obesity due to elevated inflammation level (13). Another study reported a positive association between obesity and inflammation and mild changes in iron markers (57). Additionally, Tussing-Humphreys et al (2010) measured serum hepcidin in obese and non-obese premenopausal adult women and observed a higher hepcidin level among women with obesity (58). Another study evaluated serum hepcidin level and iron status and their association with BMI in a sample of children with obesity from Egypt and reported lower hemoglobin and ferritin levels and higher hepcidin level among obese children (59). Conversely, a study that investigated iron, hepcidin, and inflammation levels in 


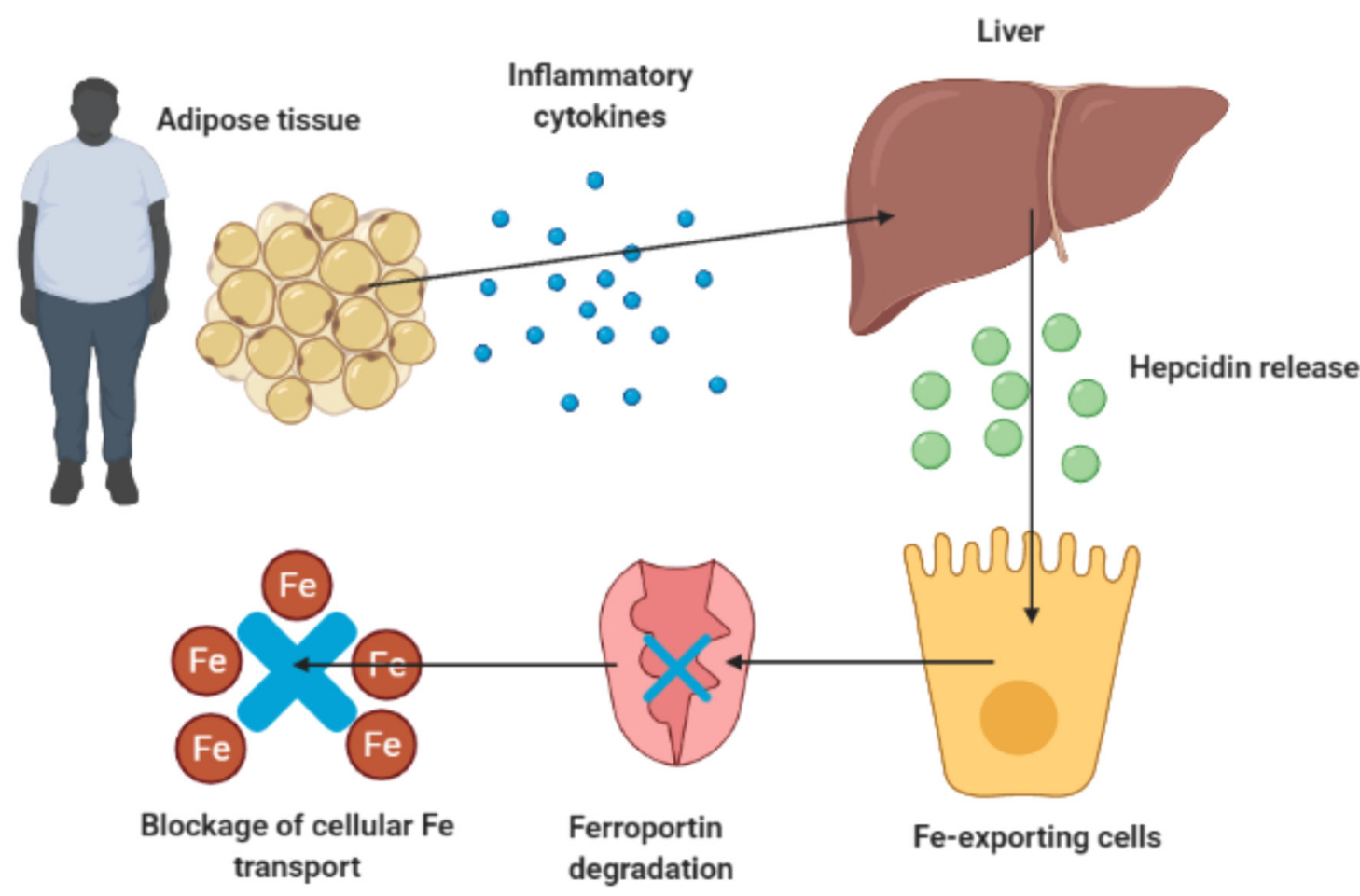

Created in BioRender.com bio

Figure 2. Role of low-grade chronic inflammation in Fe homeostasis. Increased inflammatory cytokines associated with obesity stimulate hepcidin synthesis in the liver, which blocks cellular Fe transport via ferroportin degradation.

young women with overweight and obesity concluded that iron deficiency was not linked to inflammation or hepcidin (60). Most of these reports suggested that obesity may negatively affect the iron status and cause iron deficiency due to increased serum levels of low-grade chronic inflammation and/or hepcidin.

\section{Effect of weight loss on iron status}

The traditional way to treat iron deficiency is by providing an iron supplement. However, several studies found that the efficacy of iron supplementation was significantly lower when used among individuals with overweight and obesity compared to those with normal body weight $(55,61)$. This could be caused by a reduction in iron absorption associated with elevated serum hepcidin levels observed in people with overweight and obesity (36). Weight loss that is induced by an energy-restricted diet and/or exercise may improve obesity-related hypoferremia and help to restore iron homeostasis in individuals with overweight and obesity (62). In addition, a reduction in adipose tissue is associated with alterations in the levels of pro-inflammatory cytokines, which may lead to diminished hepcidin release and improved iron status in people with overweight and obesity $(63,64)$ (Fig. 3).

A few studies investigated the effect of weight loss on iron status, low-grade chronic inflammation, and/or hepcidin release in individuals with overweight and obesity (62). Amato et al (2010) examined the effect of BMI reduction on serum hepcidin levels and iron status in obese children.
They observed a significant decrease in hepcidin and leptin levels and a significant increase in iron absorption (65). Similarly, Gong et al (2014) reported an improvement of iron status with unchanged serum ferritin concentrations and an increase in transferrin saturation after the intervention, along with an improvement of inflammatory markers (66). Coimbra et al (2017) studied the effect of an eight-month physical exercise programme on hepcidin, inflammation, and iron status in overweight and obese children and adolescents. The results showed a decrease in BMI z-score, body fat mass, CRP, interleukin-6, ferritin, hepcidin, and soluble transferrin receptor, in addition to an increase in serum iron concentration (67). Cepeda-Lopez et al (2016) evaluated the effects of fat loss after bariatric surgery on inflammation, serum hepcidin, and iron absorption. After six months of follow-up, they reported that the total body fat, interleukin-6, and hepcidin were significantly lower, whereas the iron absorption increased by $28 \%$ in iron-deficient subjects (68). Another study conducted six- and twelve-month weight loss programs (high protein diet or low protein diet: $5600 \mathrm{~kJ} /$ day) among young women (18-25 years) and revealed that an initial body weight loss of more than $10 \%$ could be associated with an improved iron status regardless of the diet type and hepcidin levels (69). Recently, Kaner et al (2019) reported changes in body weight and iron parameters of premenopausal Turkish women with overweight and obesity who participated in a weight loss program. They found a statistically significant relationship between body weight loss and CRP levels and concluded that weight loss helps to improve blood iron 


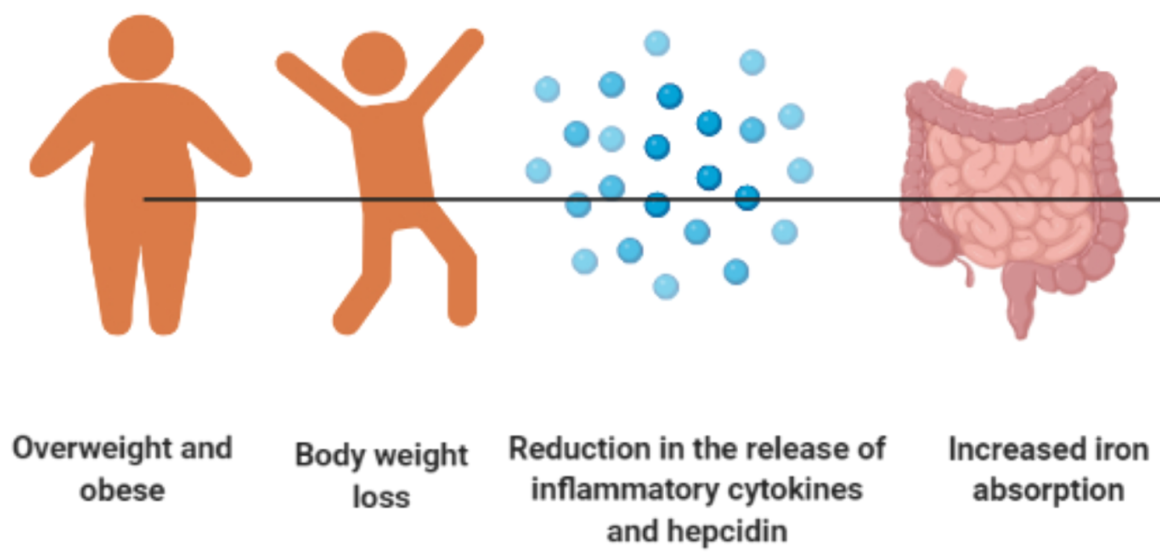

Created in BioRender.com bio

Figure 3. Effect of body weight loss on iron status. A significant weight loss in overweight and obese individuals can notably reduce the release of inflammatory cytokines and hepcidin, which increases iron absorption.

parameters due to its positive effect on low-grade chronic inflammation (70).

\section{Future research and clinical implications}

The high prevalence of obesity in combination with iron deficiency incidence observed in different age and gender groups suggesting a connection between obesity and iron status. However, few studies investigate the cause-effect relationship between them. This review highlights the importance of well-controlled randomized clinical trials to confirm the causality relationship between obesity and iron deficiency. In spite of recent evidence showing iron disturbances with obesity, outcomes from the routine iron marker, such as hemoglobin and serum ferritin, appear to be not enough to understand the causal relationship between obesity and iron status. Analysis of additional biochemical markers, including hepcidin and pro-inflammatory cytokines such as CRP and interleukin-6, should be considered. Designing energy-restricted diets that deliver sufficient iron to meet recommended intakes for subjects is essential in weight loss trials. Therefore, it would be crucial in the clinical setting to conduct a continuous assessment for dietary iron intake in subjects who received medical nutrition therapy for weight management. Moreover, physical activity levels during the weight loss trial need to be monitors and controlled. Successful weight loss of at least $5-10 \%$ of initial weight may be needed to positively impact iron, inflammation and hepcidin levels and ensure the effect of diet-induced weight loss on these markers. A strict selection criterion applied at the recruitment stage is also essential to minimize confounding effects on the weight and iron outcomes. To avoid results bias, certain groups of the target population should be excluded, such as patients with certain medical conditions, pregnant and lactating mothers, vegetarians, smokers, and those who have recently supplemented iron. Further research is needed to examine the possible connections between iron, hepcidin, and other comorbid conditions, including cardiovascular disease, diabetes and metabolic syndrome. The function of body fat amount and distribution (android vs. gynoid fat), especially concerning gender differences, should be investigated further. Finally, the link between obesity and iron deficiency is an emerging research field and one of the current limitation in the studies reviewed is the lack of formula between iron markers and obesity to predict iron deficiency.

According to the findings of this review, there is emerging evidence to support the link between obesity and iron deficiency. Increased hepcidin levels mediated by chronic inflammation are thought to be the cause of this link. The findings emphasize the significance of closely monitoring and treating iron deficiency in people with overweight and obesity. In those patients, routine iron status markers such as hemoglobin, serum ferritin, and serum iron appear to be a sound indicator of iron status. In patients with class 2 obesity and above (BMI $\geq 35$ ), caution is advised when using ferritin because inflammatory-related iron disturbances may be more noticeable. Thus, examining additional biochemical markers such as soluble transferrin receptor and hepcidin should be considered to evaluate iron status accurately. Furthermore, while attempting to determine iron status in individuals with overweight and obesity, potential confounding from higher inflammation should be considered. This could involve measuring acute phase proteins like CRP, or, at least, avoiding iron testing during periods of apparent infection. Initial assessment for iron status in patients with overweight and obesity seeking medical nutrition therapy for weight loss might be advantageous in the clinical setting. This is especially important for women who have a higher iron requirement, such as those with a history of iron insufficiency, heavy or protracted menses, or low iron intake. It is critical to design energy-restricted diets that supply sufficient iron to meet the dietary needs of overweight and obese patients. 


\section{Conclusions}

This review describes the association between obesity and iron deficiency. The mechanisms responsible for this relationship remains undefined. However, growing evidence highlights a principal role for elevation in low-grade chronic inflammation and hepcidin levels associated with obesity in disturbances in iron homeostasis, which may lead to hypoferremia. Because of the high overweight and obesity prevalence globally, it is important to develop public health strategies to manage obesity-related hypoferremia. Individuals with overweight and obesity should be targeted by periodical screening for iron status, particularly in population groups at high risk for iron deficiency anemia, including children, adolescents, and women of childbearing age. Common clinical signs and symptoms of anemia are usually not shown in patients at the early stage of iron deficiency anemia. Therefore, periodical monitoring of iron markers would be beneficial for patients with overweight and obesity. In addition, designing effective weight loss programs via an energy-restricted diet and/or exercise can help individuals with overweight and obesity to lose excessive body weight and restore iron homeostasis. Finally, future studies with robust, well-designed randomized clinical trials, relevant adjustment for important confounders, and use of various markers related to the iron status, inflammation, and hepcidin are needed to examine the putative causal relationship between obesity and iron deficiency.

\section{Acknowledgements}

Not applicable.

\section{Funding}

No funding was received.

\section{Availability of data and materials}

Not applicable.

\section{Authors' contributions}

NMA, AA and HANAJ acquired the resources by searching Scopus, PubMed and Google Scholar, collected the data from retrieved articles and drafted the initial manuscript. WMRWH reviewed and edited the manuscript for important intellectual content. Data authentication is not applicable. All authors have read and approved the final manuscript.

\section{Ethics approval and consent to participate}

Not applicable.

\section{Patient consent for publication}

Not applicable.

\section{Competing interests}

The authors declare that they have no competing interests.

\section{References}

1. Chooi YC, Ding C and Magkos F: The epidemiology of obesity. Metabolism 92: 6-10, 2019.

2. Cappellini MD, Musallam KM and Taher AT: Iron deficiency anaemia revisited. J Intern Med 287: 153-170, 2020

3. Gregg EW and Shaw JE: Global health effects of overweight and obesity. N Engl J Med 377: 80-81, 2017.

4. Camaschella C: New insights into iron deficiency and iron deficiency anemia. Blood Rev 31: 225-233, 2017.

5. Percy L, Mansour D and Fraser I: Iron deficiency and iron deficiency anaemia in women. Best Pract Res Clin Obstet Gynaecol 40: 55-67, 2017.

6. Halib H, Muda WM, Dam PC and Mohamed HJ: Prevalence of iron deficiency and its associated risk factors among primary school children in Kelantan. J Fundam Appl Sci 9: 397-412, 2017.

7. Bah F, Harith S and Farisni TN: Food knowledge and practices related to anemic conditions among pregnant women in Kuala Terengganu, Malaysia. Indonesian J Pub Health 7: 19-28, 2020.

8. Zani H, Shahril MR, Wan Abdul Rahman WN, Mukhali HB, Ismail R and Mohd Yusop Y: Anaemia-related knowledge amongst pregnant women in Kuala Terengganu, Malaysia. Asian J Med Biomed 4: 1-9, 2020.

9. Zhao L, Zhang X, Shen Y, Fang X, Wang Y and Wang F: Obesity and iron deficiency: A quantitative meta-analysis. Obes Rev 16: 1081-1093, 2015.

10. Pinhas-Hamiel O, Newfield RS, Koren I, Agmon A, Lilos P and Phillip M: Greater prevalence of iron deficiency in overweight and obese children and adolescents. Int $\mathrm{J}$ Obes Relat Metab Disord 27: 416-418, 2003.

11. Nead KG, Halterman JS, Kaczorowski JM, Auinger P and Weitzman M: Overweight children and adolescents: A risk group for iron deficiency. Pediatrics 114: 104-108, 2004.

12. Yanoff LB, Menzie CM, Denkinger B, Sebring NG, McHugh T, Remaley AT and Yanovski JA: Inflammation and iron deficiency in the hypoferremia of obesity. Int J Obes (Lond) 31: 1412-1419, 2007.

13. Kaner G, Pekcan G, Pamuk G, Pamuk B and Amoutzopoulos B: Is iron deficiency related with increased body weight? A cross-sectional study. Prog Nutr 18: 102-110, 2016.

14. Huang YF, Tok TS, Lu CL, Ko HC, Chen MY and Chen SC: Relationship between being overweight and iron deficiency in adolescents. Pediatr Neonatol 56: 386-392, 2015.

15. Egwurugwu JN, Ekweogu CN, Nwamkpa P, Ohamaeme MC, Ugwuezumba PC and Ogunnaya FU: Association between serum phosphate and iron concentrations with body mass index in a population of adults in Orlu, Imo State, Nigeria. Niger J Exp Clin Biosci 6: 1-7, 2018.

16. D'angelo G: Role of hepcidin in the pathophysiology and diagnosis of anemia. Blood Res 48: 10-15, 2013.

17. Aigner E, Feldman A and Datz C: Obesity as an emerging risk factor for iron deficiency. Nutrients 6: 3587-3600, 2014.

18. Al-Fartusie FS and Mohssan SN: Essential trace elements and their vital roles in human body. Indian J Adv Chem Sci 5: 127-136, 2017.

19. Anderson GJ and Frazer DM: Current understanding of iron homeostasis. Am J Clin Nutr 106 (Suppl 6): 1559S-1566S, 2017.

20. Milto IV, Suhodolo IV, Prokopieva VD and Klimenteva TK: Molecular and cellular bases of iron metabolism in humans. Biochemistry (Mosc) 81: 549-564, 2016.

21. Abbaspour N, Hurrell R and Kelishadi R: Review on iron and its importance for human health. J Res Med Sci 19: 164-174, 2014.

22. Dev S and Babitt JL: Overview of iron metabolism in health and disease. Hemodial Int 21 (Suppl 1): S6-S20, 2017.

23. Fuqua BK, Vulpe CD and Anderson GJ: Intestinal iron absorption. J Trace Elem Med Biol 26: 115-119, 2012.

24. Morgan EH and Oates PS: Mechanisms and regulation of intestinal iron absorption. Blood Cells Mol Dis 29: 384-399, 2002.

25. Handelman GJ and Levin NW: Iron and anemia in human biology: A review of mechanisms. Heart Fail Rev 13: 393-404, 2008.

26. Ganz T and Nemeth E: Hepcidin and iron homeostasis. Biochim Biophys Acta 1823: 1434-1443, 2012.

27. Reichert CO, da Cunha J, Levy D, Maselli LM, Bydlowski SP and Spada C: Hepcidin: Homeostasis and diseases related to iron metabolism. Acta Haematol 137: 220-236, 2017.

28. Ganz T: Hepcidin-a regulator of intestinal iron absorption and iron recycling by macrophages. Best Pract Res Clin Haematol 18: 171-182, 2005 .

29. Sangkhae V and Nemeth E: Regulation of the iron homeostatic hormone hepcidin. Adv Nutr 8: 126-136, 2017. 
30. Chaston T, Chung B, Mascarenhas M, Marks J, Patel B, Srai SK and Sharp P: Evidence for differential effects of hepcidin in macrophages and intestinal epithelial cells. Gut 57: 374-382, 2008.

31. Ellulu MS, Patimah I, Khaza'ai H, Rahmat A and Abed Y: Obesity and inflammation: The linking mechanism and the complications. Arch Med Sci 13: 851-863, 2017.

32. Fontana L, Eagon JC, Trujillo ME, Scherer PE and Klein S: Visceral fat adipokine secretion is associated with systemic inflammation in obese humans. Diabetes 56: 1010-1013, 2007.

33. Coimbra S, Catarino C and Santos-Silva A: The role of adipocytes in the modulation of iron metabolism in obesity. Obes Rev 14 771-779, 2013.

34. Nemeth E, Rivera S, Gabayan V, Keller C, Taudorf S, Pedersen BK and Ganz T: IL-6 mediates hypoferremia of inflammation by inducing the synthesis of the iron regulatory hormone hepcidin. J Clin Invest 113: 1271-1276, 2004.

35. Wrighting DM and Andrews NC: Interleukin-6 induces hepcidin expression through STAT3. Blood 108: 3204-3209, 2006

36. Stoffel NU, El-Mallah C, Herter-Aeberli I, Bissani N, Wehbe N, Obeid $\mathrm{O}$ and Zimmermann MB: The effect of central obesity on inflammation, hepcidin, and iron metabolism in young women. Int J Obes (Lond) 44: 1291-1300, 2020.

37. Gozzelino R and Arosio P: Iron homeostasis in health and disease. Int J Mol Sci 17: 130, 2016.

38. Schmidt PJ: Regulation of iron metabolism by hepcidin under conditions of inflammation. J Biol Chem 290: 18975-18983, 2015

39. Weiss G: Iron metabolism in the anemia of chronic disease. Biochim Biophys Acta 1790: 682-693, 2009.

40. David V, Martin A, Isakova T, Spaulding C, Qi L, Ramirez V, Zumbrennen-Bullough KB, Sun CC, Lin HY, Babitt JL and Wolf M: Inflammation and functional iron deficiency regulate fibroblast growth factor 23 production. Kidney Int 89: 135-146, 2016

41. Shimada T, Hasegawa H, Yamazaki Y, Muto T, Hino R, Takeuchi Y, Fujita T, Nakahara K, Fukumoto S and Yamashita T: FGF-23 is a potent regulator of vitamin D metabolism and phosphate homeostasis. J Bone Miner Res 19: 429-435, 2004

42. Agoro R, Park MY, Le Henaff C, Jankauskas S, Gaias A, Chen G, Mohammadi M and Sitara D: C-FGF23 peptide alleviates hypoferremia during acute inflammation. Haematologica 106: 391-403, 2021.

43. Agoro R, Montagna A, Goetz R, Aligbe O, Singh G, Coe LM, Mohammadi M, Rivella S and Sitara D: Inhibition of fibroblast growth factor 23 (FGF23) signaling rescues renal anemia. FASEB J 32: 3752-3764, 2018

44. Wenzel B, Stults H and Mayer J: Hypoferraemia in obese adolescents. Lancet 2: 327-328, 1962.

45. Lecube A, Carrera A, Losada E, Hernández C, Simó R and Mesa J: Iron deficiency in obese postmenopausal women. Obesity (Silver Spring) 14: 1724-1730, 2006

46. Menzie CM, Yanoff LB, Denkinger BI, McHugh T, Sebring NG Calis KA and Yanovski JA: Obesity-related hypoferremia is not explained by differences in reported intake of heme and nonheme iron or intake of dietary factors that can affect iron absorption. J Am Diet Assoc 108: 145-148, 2008.

47. Aderibigbe OR, Pisa PT, Vorster HH and Kruger SH: The relationship between iron status and adiposity in women from developing countries: A review. Crit Rev Food Sci Nutr 54: 553-560, 2014

48. Paknahad Z, Mahboob S, Omidvar N, Ebrahimi M, Ostadrahimi A and Afiatmilani SH: Body Mass Index and its relationship with haematological indices in Iranian women. Pak J Nutr 7: 377-380, 2008 .

49. Ettyang GA, van Marken Lichtenbelt WD, Oloo A and Saris WH: Serum retinol, iron status and body composition of lactating women in Nandi, Kenya. Ann Nutr Metab 47: 276-283, 2003

50. Famodu AA and Awodu OA: Anthropometric indices as determinants of haemorheological cardiovascular disease risk factors in Nigerian adults living in a semi-urban community. Clin Hemorheol Microcirc 43: 335-344, 2009.

51. Aderibigbe OR, Pisa PT, Mamabolo RL, Kruger HS, Vorster HH and Kruger A: Iron status and cardiovascular disease risk in black South African women: The PURE study. South African J Clin Nutr 24: 179-185, 2011.

52. Eckhardt CL, Torheim LE, Monterrubio E, Barquera S and Ruel MT: The overlap of overweight and anaemia among women in three countries undergoing the nutrition transition. Eur J Clin Nutr 62: 238-246, 2008.
53. Kordas K, Fonseca Centeno ZY, Pachón H and Jimenez Soto AZ: Being overweight or obese is associated with lower prevalence of anemia among Colombian women of reproductive age. J Nutr 143: 175-181, 2013.

54. Cheng HL, Bryant C, Cook R, O'Connor H, Rooney $\mathrm{K}$ and Steinbeck K: The relationship between obesity and hypoferraemia in adults: A systematic review. Obes Rev 13: 150-161, 2012.

55. Zimmermann MB, Zeder C, Muthayya S, Winichagoon $\mathrm{P}$ Chaouki N, Aeberli I and Hurrell RF: Adiposity in women and children from transition countries predicts decreased iron absorption, iron deficiency and a reduced response to iron fortification. Int J Obes (Lond) 32: 1098-1104, 2008.

56. Alam F, Memon AS and Fatima SS: Increased body mass index may lead to hyperferritinemia irrespective of body iron stores. Pak J Med Sci 31: 1521-1526, 2015.

57. Shekarriz R and Vaziri MM: Iron profile and inflammatory status of overweight and obese women in Sari, North of Iran. Int J Hematol Oncol Stem Cell Res 11: 108-113, 2017.

58. Tussing-Humphreys LM, Nemeth E, Fantuzzi G, Freels S, Guzman G, Holterman AX and Braunschweig C: Elevated systemic hepcidin and iron depletion in obese premenopausal females. Obesity (Silver Spring) 18: 1449-1456, 2010.

59. Abdel Hamed ER, Sallam SF, Hamdy HA, El Shafie AI, El Kassas GM, Khairy SA and Abdelsalam HM: Serum hepcidin level and iron status in a sample of obese Egyptian children. Med Res J 14: 7-11, 2015.

60. Cheng HL, Bryant CE, Rooney KB, Steinbeck KS, Griffin HJ, Petocz P and O'Connor HT: Iron, hepcidin and inflammatory status of young healthy overweight and obese women in Australia. PLoS One 8: e68675, 2013.

61. Baumgartner J, Smuts CM, Aeberli I, Malan L, Tjalsma H and Zimmermann MB: Overweight impairs efficacy of iron supplementation in iron-deficient South African children: A randomized controlled intervention. Int J Obes (Lond) 37: 24-30, 2013.

62. Teng IC, Tseng SH, Aulia B, Shih CK, Bai CH and Chang JS: Can diet-induced weight loss improve iron homoeostasis in patients with obesity: A systematic review and meta-analysis. Obes Rev 21: e13080, 2020.

63. Chang JS, Li YL, Lu CH, Owaga E, Chen WY and Chiou HY: Interleukin-10 as a potential regulator of hepcidin homeostasis in overweight and obese children: A cross-sectional study in Taiwan. Nutrition 30: 1165-1170, 2014

64. Sanad M, Osman M and Gharib A: Obesity modulate serum hepcidin and treatment outcome of iron deficiency anemia in children: A case control study. Ital J Pediatr 37: 34, 2011.

65. Amato A, Santoro N, Calabrò P, Grandone A, Swinkels DW, Perrone L and del Giudice EM: Effect of body mass index reduction on serum hepcidin levels and iron status in obese children. Int J Obes (Lond) 34: 1772-1774, 2010.

66. Gong L, Yuan F, Teng J, Li X, Zheng S, Lin L, Deng H, Ma G Sun $\mathrm{C}$ and $\mathrm{Li}$ Y: Weight loss, inflammatory markers, and improvements of iron status in overweight and obese children. J Pediatr 164: 795-800.e2, 2014.

67. Coimbra S, Catarino C, Nascimento H, Inês Alves A, Filipa Medeiros A, Bronze-da-Rocha E, Costa E, Rocha-Pereira P, Aires L, Seabra A, et al: Physical exercise intervention at school improved hepcidin, inflammation, and iron metabolism in overweight and obese children and adolescents. Pediatr Res 82 781-788, 2017.

68. Cepeda-Lopez AC, Allende-Labastida J, Melse-Boonstra A, Osendarp SJ, Herter-Aeberli I, Moretti D, Rodriguez-Lastra R, Gonzalez-Salazar F, Villalpando S and Zimmermann MB: The effects of fat loss after bariatric surgery on inflammation, serum hepcidin, and iron absorption: A prospective 6-mo iron stable isotope study. Am J Clin Nutr 104: 1030-1038, 2016.

69. Cheng HL, Griffin HJ, Bryant CE, Rooney KB, Steinbeck KS and O'Connor HT: Impact of diet and weight loss on iron and zinc status in overweight and obese young women. Asia Pac J Clin Nutr 22: 574-582, 2013.

70. Kaner G, Pekcan AG and Şarer Yürekli BP: Effect of a weight loss intervention on iron parameters in overweight and obese Turkish women. Prog Nutr 21: 50-56, 2019.

This work is licensed under a Creative Commons Attribution-NonCommercial-NoDerivatives 4.0 International (CC BY-NC-ND 4.0) License. 